\title{
SOP Intubation des Intensivpatienten
}

Björn Ellger, Julian Bösel

\section{Einleitung zur Rubrik}

In dieser Rubrik stellen wir Standard Operating Procedures (SOPs) für häufige, intensivmedizinisch relevante Prozesse vor. Die Form ist eher im Sinne einer Schablone zu verstehen als - durchaus subjektiv gefärbte - Anregung, eigene, auf lokale Gegebenheiten adaptierte stationsinterne SOPs zu entwerfen und zu implementieren.

\section{Einleitung zur SOP Intubation des Intensivpatienten}

Das Vorgehen zur Atemwegssicherung als eine der zentralen Interventionen in der Intensivmedizin ist z. B. durch eine S1-Leitlinie der DGAI beschrieben [1]. Die endotracheale Intubation ist eine der häufigsten Interventionen auf der Intensivstation, allerdings sind auch Komplikationen in über $25 \%$ der Fälle zu beklagen. Hypoxie, Hypotension, Fehlintubation und Aspiration sind hier zu nennen [2]. Atemwegsbezogene Komplikationen machen $20 \%$ aller Komplikationen auf der Intensivstation aus, meist durch das Vorliegen eines schwierigen Atemwegs. Die Intubation des Intensivpatienten ist gegenüber der eher elektiven Intubation im OP mit wesentlich mehr Fehlerquellen und Risiken behaftet und daher eine Herausforderung. Viele Aspekte zur Intubation des Intensivpatienten sind außerdem nicht gut in Studien untersucht, und die Übertragbarkeit derselben aus dem OP ist fragwürdig. Einige Maßnahmen zur Vermeidung von Komplikationen haben sich als sinnvoll erwiesen, besonders das Einführen eines Behandlungsbündels aus Einzelmaßnahmen hat Erfolge gezeigt [3]. Ein solches Bündel für das Vorgehen bei der Intubation des erwachsenen Intensivpatienten stellen wir in dieser SOP dar, es ist auf den prinzipiell aspirationsgefährdeten Intensivpatienten ausgerichtet.

\section{Erläuterungen zur SOP}

1. Indikationen: Die Indikation zur endotrachealen Intubation ist sorgfältig zu stellen. Alternativen wie nicht-invasive Beatmung oder nasales high-flowCPAP können sinnvoll sein. Auch ist die Einwilligung des Patienten einzuholen bzw. bei reduzierter Einwilligungsfähigkeit sein mutmaßlicher Wunsch zu eruieren und zu dokumentieren.

2. Das Nüchternheitsgebot gilt bei elektiven Operationen, um das Aspirationsrisiko bei Narkoseinduktion zu senken. Kritisch kranke Patienten sind, wie der Auflistung zu entnehmen ist, in aller Regel „nicht nüchtern“ und deshalb stark aspirationsgefährdet. Daher sollte die Intubation als Rapid Sequence Induction (RSI), also mittels direkt zügig aufeinander folgend injizierter Medikamente und ohne Maskenzwischenbeatmung, durchgeführt werden. Wie genau diese RSI umgesetzt werden soll, ist Gegenstand fortwährender Diskussionen [4], wir geben hier nur eine mögliche Lösung wieder.

3. Der schwierige Atemweg ist bei kritisch kranken Patienten in etwa 10\% der Fälle anzunehmen. Wenn dies vorhersehbar ist (Dokumentation in früheren Narkoseprotokollen, Scores, klinische Tests), dann sollte hier primär eine SOP zum schwierigen Atemweg, wie sie jede Intensivstation vorhalten sollte, angewandt und z.B. die fiberoptische Wachintubation angestrebt werden [5].

4. Klare Kommunikation ist essenziell! Zunächst müssen klar die Entscheidung zur Intubation angesagt und die Aufgaben unmissverständlich zugewiesen werden. Die Vorbereitung kann in 3 Aufgabenfelder gegliedert werden. Dies geschieht durch den Teamleader, der in der Regel der erfahrenste Facharzt ist.

Wenn Sie selbst in Ihrer Klinik eine SOP haben, die auch anderen Kollegen nützlich sein könnte, freuen wir uns über eine kurze E-Mail an intensiv-u2d@thieme.de. 


\section{Intubation des Intensivpatienten bei Aspirationsgefahr'}

Risiken für Aspiration ${ }^{2}$

- (Sub-)lleus/Erbrechen

- Gastroparese

- Blutungen oberer GI-Trakt

- hoher intraabdom. Druck

- akutes Abdomen

- (Poly-) Trauma

- Notfalleingriff

- starke Angst/Stress

- schwere Atemstörung

- Schock

- Bewusstseinsstörung

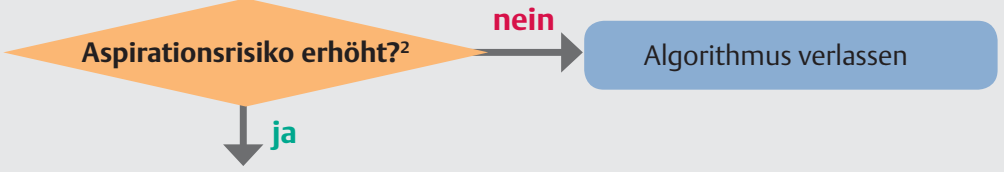

Kein Anhalt für schwierigen Atemweg? SOP „schwieriger Atemweg“

$$
\downarrow \text { ja }
$$

Entscheidung Rapid Sequence Induction (RSI)

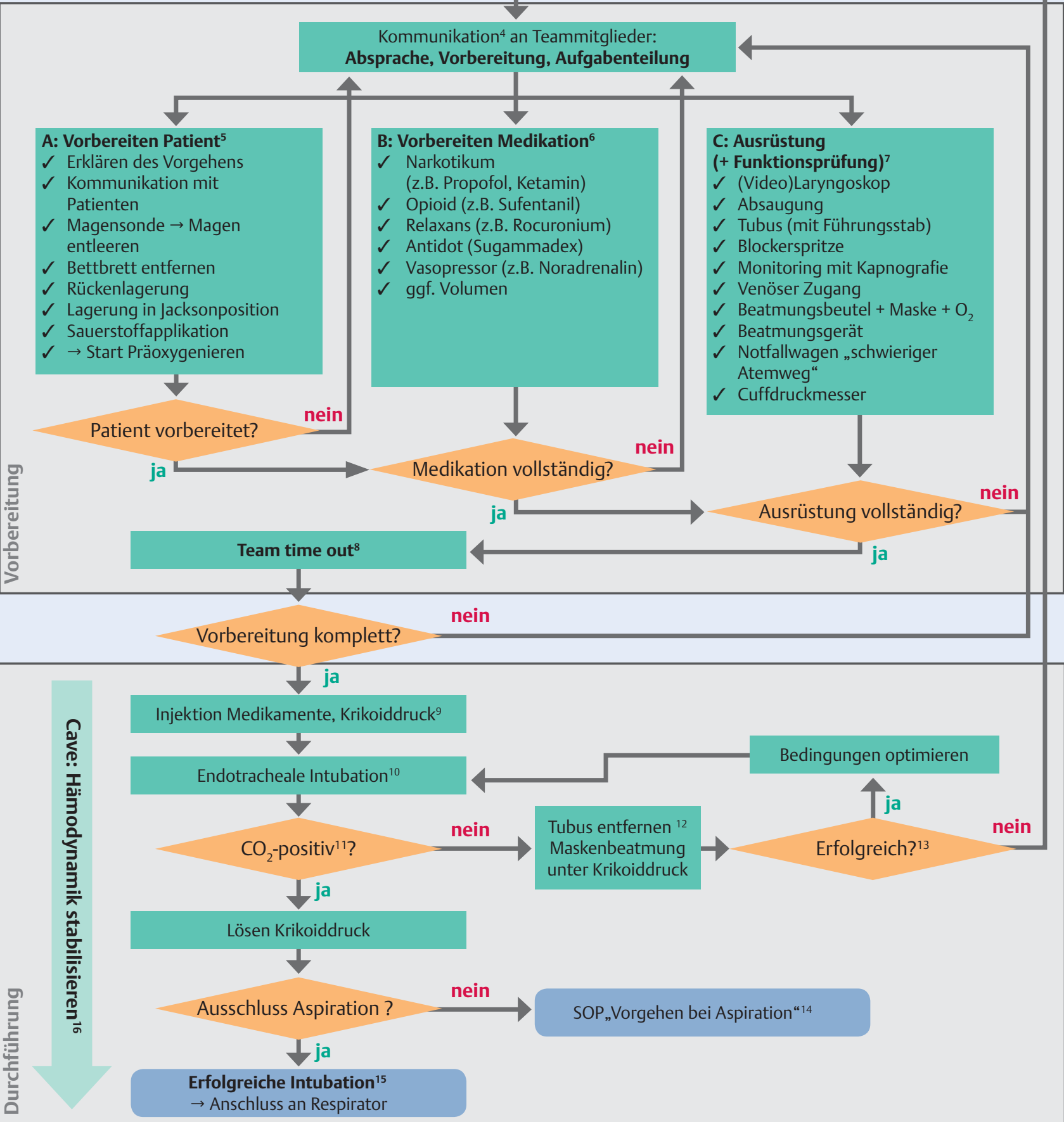


Bei diesem Teamleader laufen alle Fäden zusammen und er behält die Übersicht, ähnlich wie in einer Reanimationssituation. Es muss sichergestellt werden, dass jeder im Team die zugewiesene Aufgabe verstanden hat. Hier bietet sich die 2-Wege-Kommunikation als Strategie an. Dabei wiederholt der Empfänger die wichtigsten Anteile der Botschaft des Senders. So können Missverständnisse oder Fehler vermieden werden.

Auch muss sichergestellt sein, dass ausreichend Personal (Anzahl und Ausbildung) vorhanden ist, bei Hochrisikopatienten empfiehlt sich die Anwesenheit von 2 ausgebildeten Ärzten und mind. 2 geschulten Pflegekräften.

5. Vorbereitung Patient: Der Patient ist in der Regel einem hohen Stress ausgesetzt. Beruhigen und empathische Zuwendung sind hier essentiell. Das Absaugen von Mageninhalt über eine Sonde ist sinnvoll. Die Sonde sollte aber zur Intubation entfernt werden (Aspirationsschiene). Die Rückenlage, ggf. mit leicht erhöhtem Oberkörper (ca. $30^{\circ}$, Cave: Patient kann herunterrutschen), und Kopflagerung in Jackson-Position gestattet meist optimale Intubationsbedingungen. Dass eine Lagerung mit erhöhtem Oberkörper die Regurgitation verhindert, ist sehr zweifelhaft und wahrscheinlich erst bei einer Lagerung mit $>45^{\circ}$ zu erwarten; bei so extremer Lagerung kann aber das Intubieren erschwert werden und wir empfehlen darauf zu verzichten. Für den Fall einer Regurgitation sollte sofort eine Trendelenburglagerung durchführbar sein. Die Präoxygenierung (und Denitrogenisierung) ist wichtig um die Apnoetoleranz zu verlängern. Dies kann über eine dicht sitzende Maske oder auch über (nasales) High-flow CPAP erfolgen. Das Bett sollte im Zimmer so positioniert werden, dass es rundherum erreichbar ist (vor allem Kopfende zur Intubation). Um den Zugang zum Kopf zu verbessern sollten Begrenzungen oder Bügel am Kopfende des Bettes entfernt werden.

6. Vorbereitung Medikation: Die ideale Medikation ist unbekannt, und einzelne Substanzen sind in ständiger Diskussion. Als Narkotikum erscheint Etomidate aus verschiedenen Gründen eher ungeeignet [6], dagegen werden Propofol, Midazolam, Ketamin und Thiopental in jeweils individuell adaptierter Dosierung (,just enough to do the job“, aber auch nicht weniger) häufig verwendet. Ein Opioid (z. B. Remifentanil, Sufentanil) dämpft die Schmerzreaktion und kann ggf. schädigende Tachykardien vermeiden helfen. Als Relaxans erscheint Rocuronium zur Zeit die beste Alternative, vor allem aufgrund seiner schnellen Anschlagszeit, seinem geringen Risikopro- fil und der Antagonisierbarkeit mit Sugammadex [7]. Da das Succinylcholin mit verschiedenen Risiken (z.B. Hyperkaliämie) beim kritisch Kranken einhergeht, scheint dieses Medikament eher ungeeignet. Es empfiehlt sich, Volumen und einen Vasopressor (möglichst als Perfusor, Leitung und Zugang vorgespült) an den Patienten anzuschließen, um Hypotensionen nach Narkoseinduktion zügig behandeln zu können. Alle Medikamente müssen bereitliegen, die Spritzen müssen entsprechend beschriftet sein.

7. Ausrüstung: die Fachgesellschaften haben klare Empfehlungen für die Ausstattung von Arbeitsplätzen auf Intensivstationen gegeben. Diese Ausrüstung muss vollständig und funktionsgeprüft vorhanden sein. Hämodynamisches Monitoring muss angeschlossen und funktionstüchtig sein, dies gilt vor allem für die Kapnografie! Da ein schwieriger Atemwege oft zu erwarten ist, muss die entsprechende Ausrüstung (z. B. Device für einen supraglottischen Luftweg) in Reichweite sein.

Es ist sinnvoll, wenn alle in einer Institution standardmäßig verwendeten Instrumente in einer „Interventionsbox“ zusammengepackt sind. Dies verhindert das hektische Zusammensuchen der notwendigen Materialien in einer Stresssituation und erhöht die Sicherheit (Zeitgewinn!).

8. Team Time Out: Vor Injektion der Medikamente halten alle kurz inne, und es erfolgt die endgültige Absprache vor Beginn der Intubation. Es wird sichergestellt, dass alle Teammitglieder sich und ihre Qualifikation kennen (kurzes Vorstellen). Das Team wird auf einen gemeinsamen Stand gebracht, und es kann sichergestellt werden, dass alle Vorbereitungen vollständig abgeschlossen wurden und jedem seine Aufgabe und der Ablauf klar ist. Das Team Time Out führt der Teamleader durch, hier ein Beispiel:

a. „Wir machen eine Notfallintubation bei Herrn X wegen Hypoxie, ein schwieriger Luftweg ist nicht zu erwarten, die Hämodynamik ist stabil. AirwayWagen vor Ort? Das Intubationszubehör ist vollständig, und ich habe die Funktion geprüft, Absaugung läuft.“

b. „Ich präoxygeniere schon, am Kopf ist alles vorbereitet, der Patient ist gelagert. Ich mache die Intubation."

c. Zu Pflegekraft A: „Sind die Medikamente vollständig? Du injizierst gleich, wenn ich es ansage.“

d. Pflegekraft B: „Monitoring vollständig und Beatmungsgerät bereit? Du machst Krikoiddruck.“

e. Zum zweiten Arzt: „Du kümmerst dich um das Monitoring und das Stabilisieren der Hämodynamik.“

f. „Alles klar? Wir beginnen ...“ 
Die Teammitglieder bestätigen jeden Schritt, bei direkter Aufgabenverteilung z. B. auch durch 2-Wege-Kommunikation.

9. Die Injektion der Medikamente inklusive Relaxans erfolgt schnell nacheinander, der Teamleader sagt die exakte Dosierung an. Bei Erlöschen der Schutzreflexe scheint der Krikoiddruck (Sellick-Manöver), richtig ausgeführt, vorteilhaft zu sein [4], auch wenn die Evidenzlage sehr dürftig ist. Problematisch kann eine durch den Krikoiddruck erschwerte Intubation sein sowie das Auslösen von Husten oder Erbrechen bei zu früher Applikation. Sollte es unter Krikoiddruck zu aktivem Erbrechen kommen, so ist der Druck wegen der Gefahr einer Ösophagusruptur sofort zu lösen.

Sollte eine Rückkehr zur Spontanatmung notwendig sein, so kann ggf. mit Sugammadex antagonisiert werden. Die Diskussion um dieses Vorgehen ist aber in aller Regel eher akademisch, da aufgrund der verabreichten Analgosedierung und des klinischen Zustandes, der die Intubationsindikation determiniert, eine zeitnahe Rückkehr zur suffizienten Spontanatmung nicht erwartet werden darf.

10. Intubation: Hier erscheint der primäre Einsatz der Videolaryngoskopie sinnvoll [8], vor allem, wenn die Prozedur durch Ärzte in Weiterbildung durchgeführt wird. Bei der Verwendung eines Führungsstabes sind auf eine atraumatische Technik und frühes Zurückziehen des Stabes zu achten. Der Cuff muss vorsichtig insuffliert werden, ein Überblähen kann akute Trachealverletzungen verursachen und ist vielleicht noch gefährlicher als ein Führungsstab.

11. Die Tubuslage wird immer per exspiratorischem $\mathrm{CO}_{2}$-Signal verifiziert. Erst bei positivem Signal wird der Krikoiddruck gelockert. Anschließend kann durch Auskultation die Tubuslage weiter optimiert werden. Ein Verzicht auf die $\mathrm{CO}_{2}$-Kontrolle ist obsolet. Bei Zweifel: Tubus entfernen („When in doubt, take it out“).

12. Sollte eine primäre Intubation nicht glücken, so kann auch eine Maskenbeatmung, möglichst unter erhaltenem Krikoiddruck, durchgeführt werden und dann nach Optimieren von Intubationsbedingungen (z.B. Lagerung optimieren, Optimieren der Narkose) ein erneuter Versuch unternommen werden.
13. Sollten 2 Intubationsversuche nicht geglückt sein, so muss die SOP zum schwierigen Atemweg begonnen werden. Oft hilft der Intubationsversuch durch einen erfahreneren Arzt, denn vielfaches Probieren und Stochern durch den unerfahreneren kann deletäre oder fatale Folgen haben.

14. Bei Regurgitation relevanter Flüssigkeitsmengen muss verhindert werden, dass diese in die Lunge laufen. Die Trendelenburglagerung stellt dies sicher. Sollte es während des Intubationsversuches zu einem solchen Ereignis kommen, so muss unmittelbar das Sekret abgesaugt werden. Die Platzierung des Endotrachealtubus in der Trachea dichtet diese ab. Es ist sinnvoll auch das Vorgehen bei Aspiration in einem Standard zu regeln. Eine bronchoskopische Sekretentfernung ist sicher sinnvoll. Blindes Spülen führt zur Verteilung des Sekretes in der Lunge und muss unterbleiben. Die Diskussion um eine antibiotische Therapie oder protektive Beatmung soll hier nicht begonnen werden. Die Behandlung richtet sich prinzipiell nach dem Ausmaß der Aspiration [9].

15. Nach Verifizieren der Tubuslage erfolgt der Anschluss ans Beatmungsgerät und das Fixieren des Tubus. Es folgt der Beginn einer Analgosedierung und das Einstellen einer protektiven Ventilation (Verweis auf up2date SOP protektive Ventilation) sowie eine Cuffdruckmessung. Wichtig ist, dass die nun eingeleitete kontrollierte Beatmung nicht als Therapie an sich aufgefasst wird. Die zur respiratorischen Dekompensation führende Ursache muss zwingend behandelt werden.

16. Nach Induktion der Narkose oder nach Intubation, wenn der Stress des Patienten nachlässt, kommt es häufig zu Blutdruckabfällen. Ein zweiter Arzt sollte mit Volumen und Vasopressoren dem entgegen wirken, um einer kritischen Hypotension vorzubeugen. 


\section{Über die Autoren}

\section{Björn Ellger}

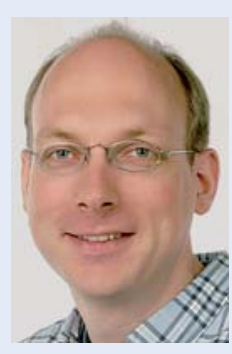

\section{Julian Bösel}

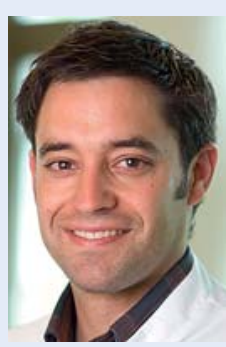

Prof. Dr. med. Björn Ellger ist Leiter der operativen Intensivstationen des Universitätsklinikums Münster.

E-Mail: ellger@anit.uni-muenster.de

PD Dr. med. Julian Bösel ist Oberarzt der neurologischen Intensivstation des Universitätsklinikums Heidelberg. E-Mail: julian.boesel@med.uniheidelberg.de

\section{Weiterführende Literatur}

1 Piepho T, Cavus E, Noppens R et al. S1 Leitlinie: Atemwegsmanagement. AWMF-Register Nr. 001/028. 2015

2 Griesdale DE, Bosma TL, Kurth T et al. Complications of endotracheal intubation in the critically ill. Intensive Care Med 2008; 34: 1835 - 1842

3 Jaber S, Jung B, Corne P et al. An intervention to decrease complications related to endotracheal intubation in the intensive care unit: a prospective, multiple-center study. Intensive Care Med 2010; 36: 248-255

4 El-Orbany M, Connolly LA. Rapid sequence induction and intubation: current controversy. Anesth Analg 2010; 110 : 1318-1325

5 Theisen MM, Enk D, Meißner A et al. Airway Management in der Intensivmedizin. Intensivmedizin up2date 2015; 11 : $13-31$

6 van den Heuvel I, Wurmb TE, Böttinger BW et al. Pros and cons of etomidate - more discussion than evidence? Current Opinion in Anesthesiology 2013; 26: 404-408

7 Luxen ], Trentzsch H, Urban B. [Rocuronium and sugammadex in emergency medicine: requirements of a muscle relaxant for rapid sequence induction]. Anaesthesist 2014; 63: 331 - 337

8 Karalapillai D, Darvall J, Mandeville J et al. A review of video laryngoscopes relevant to the intensive care unit. Indian J Crit Care Med 2014; 18: 442-452

9 Abdulla S. Pulmonary aspiration in perioperative medicine. Acta Anaesthesiol Belg 2013; 64: 1 - 13

Alle bisher publizierten SOPs der Intensivmedizin up2date finden Sie online unter www.thieme.de/ intensiv-u2d/sops. 